\title{
Comparative study of degradation effects in different shape memory alloy systems
}

\author{
A.G. Mayer, H. Scherngell and A.C. KneissI \\ Institute of Physical Metallurgy and Materials Testing, University of Leoben, Franz-Josef-Str. 18, \\ 8700 Leoben, Austria
}

\begin{abstract}
The presented investigations aim at clarifying the influence of microstructure and applied training procedure on the stability of the created intrinsic two-way shape memory effect (TWSME). The investigations are carried out on three different alloy systems in order to get an appropriate variation of the microstructure with respect to dislocation density, second phase particles and grain size. A uniaxial TWSME is induced by a thermomechanical treatment in wire specimens of the alloys $\mathrm{NiTi}, \mathrm{CuAlNi}$ and a two phase alloy composed of a NiTi matrix and dispersoids of tungsten. Subsequently, thermal cycles are carried out, continuously observing the changes in the deformation behaviour.

The results suggest that the training must result in both, a large pseudoplastic strain as well as a pronounced irreversible strain in order to obtain a TWSME of high magnitude. The degradation of the TWSME in the NiTibased alloys was found to consist of two characteristic stages, which can always be recognised, independent of the preliminary heat treatment. The obvious strengthening effect of the particles in the two-phase alloy NiTi-W has a very positive effect on the stability of the created TWSME. This effect can even be improved by preliminary cold working. The stability of the effect in the CuAlNi alloy is far better then in the binary NiTi alloy. No dramatic change in the degradation rate is observed throughout the investigated period of 5000 thermal cycles.
\end{abstract}

\section{INTRODUCTION}

One of the most promising fields for the application of shape memory alloys (SMAs) is the actuator technology. With shape memory actuators complicated movements can be realised using simple elements, free of mechanical joints, thus facilitating miniaturisation and improving reliability. The use of shape memory elements as actuators requires a cyclical application of the shape memory effect, electrically or thermally triggered. Especially in the case of strict design based tolerances, functional stability is a major concern in these applications, since it directly determines the life time of the element. The transformation temperatures, the hysteresis and in particular the magnitude of the effect must meet the given requirements and should be independent of the number of service cycles.

At present, cyclical shape changes based on the shape memory effect are mainly realised by the extrinsic two-way shape memory effect (TWSME) where the change between two pre-set shapes is obtained by constructive means. This solution has the disadvantage that for designing the element, the external force - usually a steel spring - has always to be taken into consideration, thus restricting the choice of the shape change and impeding the miniaturisation of the element. Consequently, for the design of micro-actuators, it would be more reasonable to apply the intrinsic TWSME. Contemporary research and development is concentrating very much on actuators of ever smaller sizes. The smaller the size, the more important the advantage of the intrinsic TWSME will become since it is not limited to any single mode. Since no resetting force has to be considered in design, one can truly choose from a variety of shapes and configurations.

It is the aim of this study to investigate the relation between the intrinsic TWSME effect, microstructural aspects and the stability of the effect on cyclic application. This special effect represents certainiy the most sophisticated deformation phenomenon that can be distinguished in shape memory materials, though also the most difficult to establish and to control. It is an inherently weak effect, relying exclusively on a specific substructure, thus being most sensitive to the slightest irreversible 
microstructural changes, which we suspect to be the core mechanism of functional fatigue for every type of shape memory performance.

It is particularly true for shape memory alloys that the material properties can be efficiently adjusted by optimising the microstructure. Therefore, systematic investigations will be carried out on a variety of initial microstructures in order to elaborate a comprehensive picture of this topic. Though the stability of a shape memory element ought to be referred to various functional properties, the present study will be limited to the magnitude of the intrinsic TWSME.

Preliminary investigations [1] have shown that a promising approach to improve the stability of the effect size is to increase the intensity of internal stresses. This can be achieved by appropriate microstructural design, i.e.:

increase of the amount of phase boundaries

increase of the density of dislocations

taking advantage of different coefficients of thermal expansion of two phases

increase of internal friction.

Since it is to be expected that each of these measures will have opposite effects on shape memory capability and shape memory stability of a material, it is quite probable that the microstructure representing the best compromise is a combination of the different features.

\section{EXPERIMENTAL}

In order to enable a systematic variation of the microstructure with respect to dislocation density, second phase particles and grain size, the investigations have been carried out on three different alloy systems: binary $\mathrm{NiTi}$, a dual-phase alloy consisting of a NiTi matrix containing dispersoids of tungsten and finally on a CuAlNi-based alloy. The nominal composition of the alloys as well as the preliminary heat treatments the samples have been subjected to are summarised in table 1. For each alloy the shape of the as-received material consisted in wire material with a diameter of $3 \mathrm{~mm}$. The NiTi-based alloys were hot extruded and reduced to the final diameter by stepwise cold drawing with intermediate annealing. The remaining cold-work in the as-received material resulting from the last step of cold drawing was $13 \%$. The CuAlNi wire was hot extruded till its final shape due to its poor ductility.

Specimens A2 and B2 of the NiTi-based alloys were annealed at $550^{\circ} \mathrm{C}$ in order to create a condition with a recovered microstructure as a comparison to the cold worked, as-received conditions Al, Bl. Specimens of alloy $\mathrm{C}$ were subjected to a betatizing-treatment, a precondition to observe the shape memory effect in Cu-based alloys. The duration of betatizing was varied between specimen B1 and B2 in order to establish two different grain sizes in the corresponding microstructures. The annealing after beta quenching specified in table 1 has been carried out, since preliminary investigations [2] showed that such an annealing improves the shape memory potential of the alloy. The transformation temperatures of all specimens were above room temperature.

A uniaxial two-way shape memory effect has been induced in wire specimens by a thermomechancial training treatment. The wire specimens were thermally cycled through the temperature range of phase transformation under a constant load that had been applied at room temperature. Heating was done by direct passage of current, cooling with pressurised air. After training, the shape change upon free thermal cycling was determined. The intrinsic TWSME was executed 4000 to 5000 times, continuously observing the changes in the deformation behaviour. The influence of work hardening, grain size and high internal stress on the development and the stability of the intrinsic TWSME is discussed, supported by microstructural investigations.

\section{RESULTS AND DISCUSSION}

The W-dispersoids in alloy B were introduced in order to improve the functional stability of the intrinsic TWSME by increasing the internal stress, thus impeding irreversible processes from occurring during thermal transformation cycling. We chose $\mathrm{W}$ since it is thermally stable and will hopefully not affect 
negatively the corrosion resistance of binary NiTi. In order to fabricate the ternary alloy $\mathrm{B}$, a mother alloy consisting of the elements $\mathrm{Ti}$ and $\mathrm{W}$ was produced in the first step and afterwards the corresponding amount of $\mathrm{Ni}$ was added in a remelting process. Figure 1, left, shows that at room temperature the microstructure of alloy $\mathrm{B}$ is characterised by a martensitic matrix. In order to make the size and distribution of the W-rich dispersoids become apparent, a surface layer etching was applied, resulting in micrographs like the one shown in Figure 1, right.
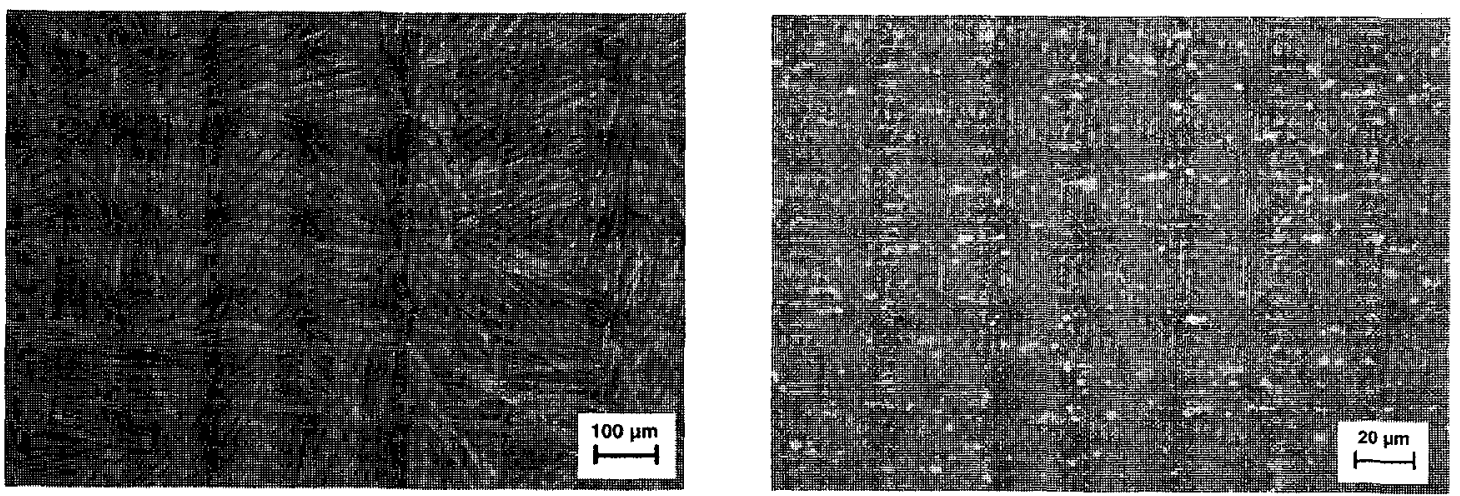

Figure 1: Left: martensitic microstructure of specimen B2; right: size and distribution of W-dispersoids in specimen B2.

Figure 2 shows optical micrographs of the microstructure of the specimens $\mathrm{C} 1$ and $\mathrm{C} 2$. A very large variation in grain size has been obtained. Whilst the mean grain size in specimen $\mathrm{Cl}$, betatized for only a short time, is only 70 microns, in specimen C2 it reaches 500 microns. The microstructure is completely martensitic and free of precipitates. This confirms that no eutectoidal dissociation of $\beta$ into the equilibrium phases did occur during the post-quench annealing at $300^{\circ} \mathrm{C}$.
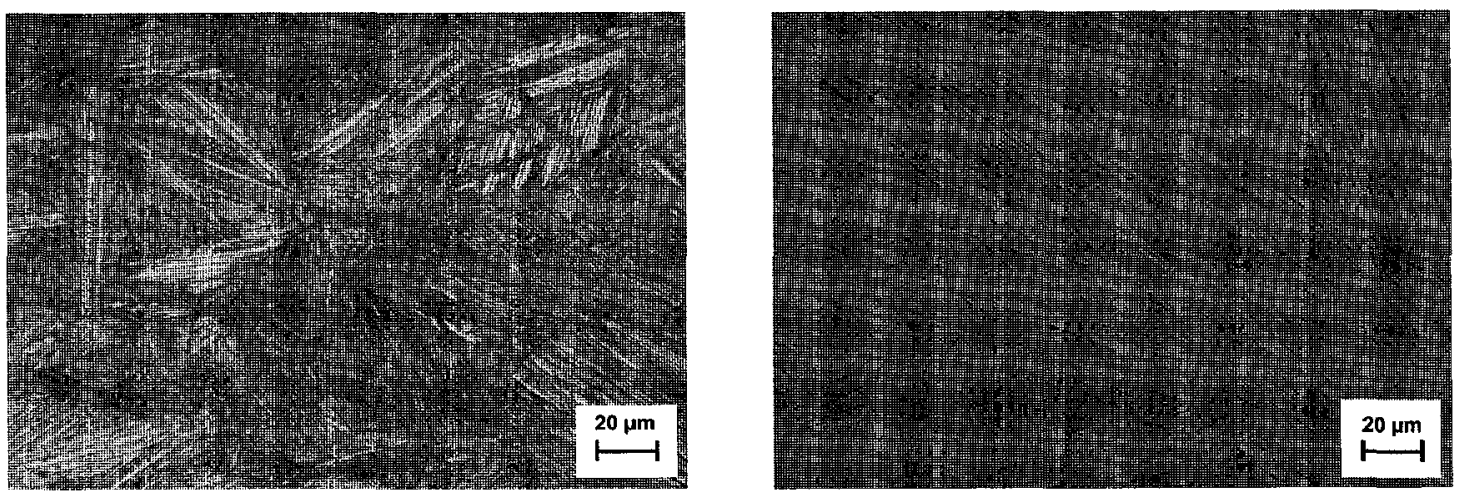

Figure 2: Microstructure of specimen $\mathrm{Cl}$, left and $\mathrm{C} 2$, right.

It is widely accepted that the creation of the intrinsic TWSME is associated with the development of irreversible strain during the thermomechanical training treatment. Some sort of asymmetry in the microstructure (e.g. retained martensite [3]) or in the submicrostructure (e.g. dislocation structures $[3,4]$ ) have to be introduced for obtaining a spontaneous shape change during cooling. In order to make sure that sufficient ductility is available in alloy $B$ to introduce the required irreversible deformation, tensile tests were carried out. Moreover, by comparing the stress-strain behaviour in the martensitic modification of alloy $\mathrm{A}$ and $\mathrm{B}$, the influence of the dispersoids on the shape memory behaviour can be deduced. Figure 3 shows a comparison of the shape of the martensite plateau of two samples with identical thermomechanical history, but only one of them containing W-dispersoids. It is apparent that the Wdispersoids, which represent a second phase not participating in the martensitic transformation, do not essentially affect the microstructural processes of pseudoplastic deformation. The dispersoids seem not to hinder effectively the formation and growth of martensite variants. The fact, that the extension and 
inclination of the martensite plateau are almost identical in specimen A2 and B2 suggests that the elastic strains originating from the mismatch between the matrix and the dispersoids are mainly accommodated by matrix twinning. A slight decrease of the maximum pseudoplastic strain occurs, evident from the smaller extension of the plateau of alloy B in Figure 3 . This is thought to be a consequence of the fact that in alloy $B$ the volume of martensite that can contribute to the transformation strain is smaller due to the dispersoids. A further quite interesting detail evident in Figure 3 is that the critical stress for martensite reorientation is smaller in the alloy containing the dispersoids. This is rather surprising since the dispersoids represent obstacles to the reorientating martensitic structure.

Table 1: Nominal composition and heat treatment of investigated samples.

\begin{tabular}{ccl}
\hline specimen & nominal composition [at\%] & \multicolumn{1}{c}{ heat treatment } \\
\hline $\mathrm{A} 1$ & $\mathrm{Ni}-50.3 \mathrm{Ti}$ & $13.5 \% \mathrm{cw}$ \\
$\mathrm{A} 2$ & $\mathrm{Ni}-50.3 \mathrm{Ti}$ & $550^{\circ} \mathrm{C} / 20^{\prime} / \mathrm{WQ}$ \\
$\mathrm{B} 1$ & $\mathrm{Ni}-50.3 \mathrm{Ti}-2 \mathrm{~W}$ & $13.5 \% \mathrm{cW}$ \\
$\mathrm{B} 2$ & $\mathrm{Ni}-50.3 \mathrm{Ti}-2 \mathrm{~W}$ & $550^{\circ} \mathrm{C} / 20^{\prime} / \mathrm{WQ}$ \\
$\mathrm{C} 1$ & $\mathrm{Cu}-25.8 \mathrm{Al}-3.6 \mathrm{Ni}$ & $800^{\circ} \mathrm{C} / 90^{\prime} / \mathrm{WQ}$ \\
& & $300^{\circ} \mathrm{C} / 120^{\circ} / \mathrm{WQ}$ \\
$\mathrm{C} 2$ & $\mathrm{Cu}-25.8 \mathrm{Al}-3.6 \mathrm{Ni}$ & $800^{\circ} \mathrm{C} / 60^{\circ} / \mathrm{WQ}$ \\
& & $300^{\circ} \mathrm{C} / 120^{\circ} / \mathrm{WQ}$ \\
\hline
\end{tabular}

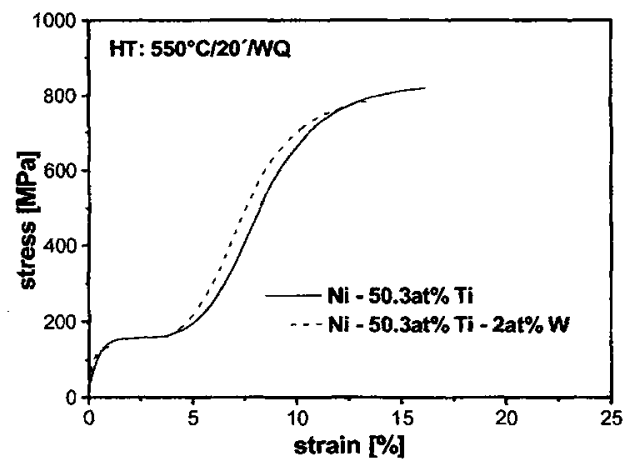

Figure 3: Comparison of the deformation behaviour of alloy $A$ and $B$.

The ductility of alloy B appears to be surprisingly good, certainly sufficient to introduce an intrinsic TWSME by thermomechanical training. In Figure 4, left, a training cycle is depicted schematically, illustrating the several particular strains involved. The wire specimen elongates on cooling, obtaining the shape $\varepsilon_{m}$ on reaching $M_{f}$ temperature. The part of the elongation that is recovered on subsequent heating corresponds to the pseudoplastic strain $\varepsilon_{\mathrm{pp}}$. Each cycle a small amount of strain, $\Delta \varepsilon_{\mathrm{p}}$, is not recovered on subsequent heating, thus changing gradually the high-temperature shape by continuously increasing the irreversible strain $\varepsilon_{\mathrm{p}}$. The micrograph in Figure 4, right, illustrates the development of the different strains as it has been recorded during thermomechanical training of specimen A2.
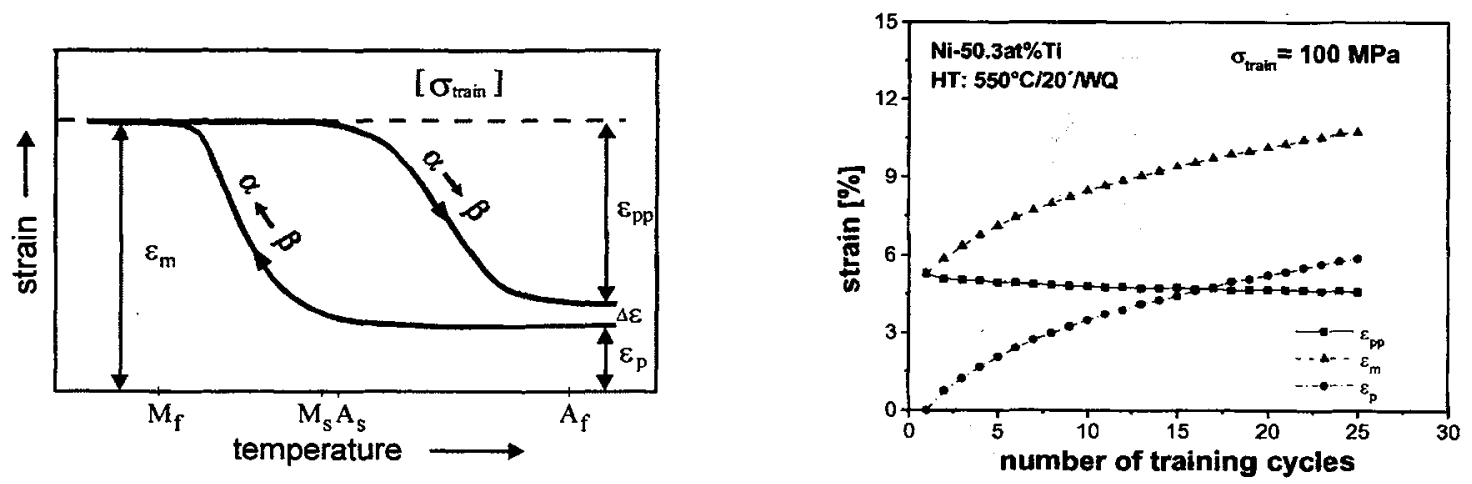

Figure 4: Left: Indication of different strains during thermomechanical training cycle, schematic curve. Right: Development of different strains during training of specimen A2.

In the case of the NiTi-based alloys, a constant stress $\sigma_{\text {train }}$ of $100 \mathrm{MPa}$ was applied, while for the CuAlNi alloy $140 \mathrm{MPa}$ were necessary to introduce an appropriate amount of true plastic deformation. In order to compare the effect of the thermomechanical training treatment on the several alloys, the different strains developed during thermomechanical training are compared in table 2 . The final values of the true plastic strain and the pseudoplastic strain accumulated throughout the training as illustrated in Figure 4, 
right, as well as the obtained two-way strain are listed. It becomes obvious that the training must result in both, a large pseudoplastic strain as well as a pronounced irreversible strain, in order to obtain a TWSME of high magnitude. From the investigations carried out with the alloys A and B one can see that coldwork affects more the development of the irreversible strain than of pseudoplastic strain. Hence it follows that a high dislocation density impedes dislocation glide more effectively than the movement of internal interfaces of twins or martensite variants.

The results from the experiments carried out with alloy $\mathrm{C}$ show the importance of the grain size on the magnitude of the developed TWSME. While the values of $\varepsilon_{\mathrm{p}}$ and $\varepsilon_{\mathrm{pp}}$ are rather the same, the size of the effect is considerably increased. This indicates that in a coarse grained microstructure the orientation of the martensite variants is far better. Due to the large grains, the univariant regions are more extensive, resulting in a larger net shape change.

Table 2: Summary of results of thermomechanical training treatments.

\begin{tabular}{cccc}
\hline specimen & $\varepsilon_{\mathrm{p}}[\%]$ & $\varepsilon_{\mathrm{pp}}[\%]$ & $\varepsilon_{2 \mathrm{w}}[\%]$ \\
\hline $\mathrm{A} 1$ & 1.9 & 3.9 & 2.0 \\
$\mathrm{~A} 2$ & 5.9 & 4.6 & 2.9 \\
$\mathrm{~B} 1$ & 1.8 & 3.5 & 0.5 \\
$\mathrm{~B} 2$ & 3.8 & 3.6 & 0.9 \\
$\mathrm{C} 1$ & 0.9 & 1.4 & 0.7 \\
$\mathrm{C} 2$ & 0.9 & 1.7 & 1.5 \\
\hline
\end{tabular}

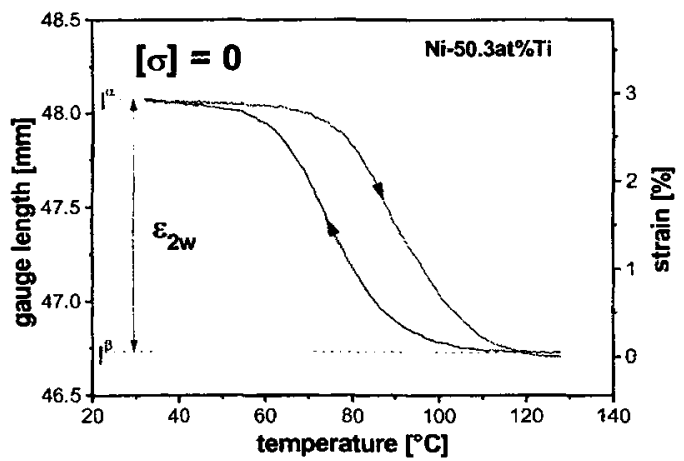

Figure 5: Two-way shape change of specimen A2 after training.
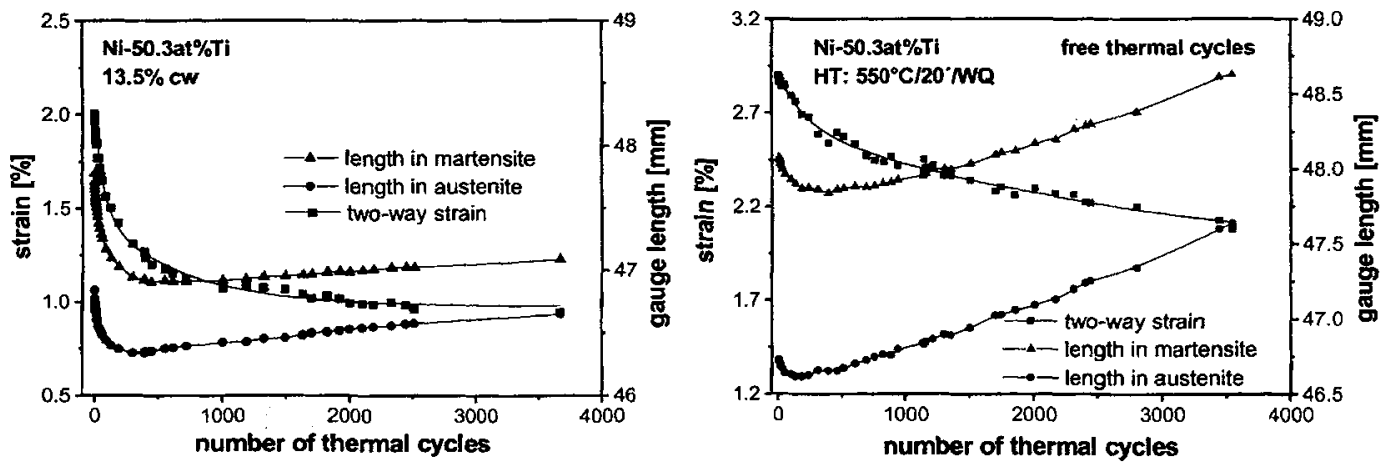

Figure 6: Change of the two-way effect due to thermal cycling; specimen A.

A two-way cycle as illustrated in Figure 5 is composed of the restoration of the original shape during heating and the deformation that accompanies cooling. Hence it follows that a degradation of the memory effect can occur in two directions: on the spontaneous shape change during the reverse transformation expressed by an increase of $1^{\beta}$ in Figure 5 - and on the trained shape change during the forward transformation - expressed by a decrease of $\left(1^{\alpha}-1^{\beta}\right)$. Consequently any change of either the hot shape $1^{\beta}$ or the cold shape $1^{\alpha}$ will directly affect the magnitude of the TWSME. Therefore in Figure 6 to Figure 8 the development of these shapes throughout the repeated application of the TWSME is illustrated together with the magnitude of the effect.

In order to achieve repeatedly the same low temperature shape $1^{\alpha}$ during stress free thermal cycling it is essential that the transformation follows the same transformation paths over and over again. The 
thermoelastic martensitic transformation occurs sequentially. Those martensite variants that have been formed first, are the last to revert. A thermodynamic balance exists at all times at the B2 / B19' interfaces between chemical and energy terms. The latter one is essentially the elastic strain energy developed as the microstructural units of new phase nucleate and grow in the original phase [5]. By this energy term, the substructure can exercise some influence on the selection of variants, that are assisted in their nucleation and growth. Consequently, some variants are favoured regarding nucleation and growth, producing the elongation $\left(1^{\alpha}-1^{\beta}\right)$ of the wire specimen during cooling.
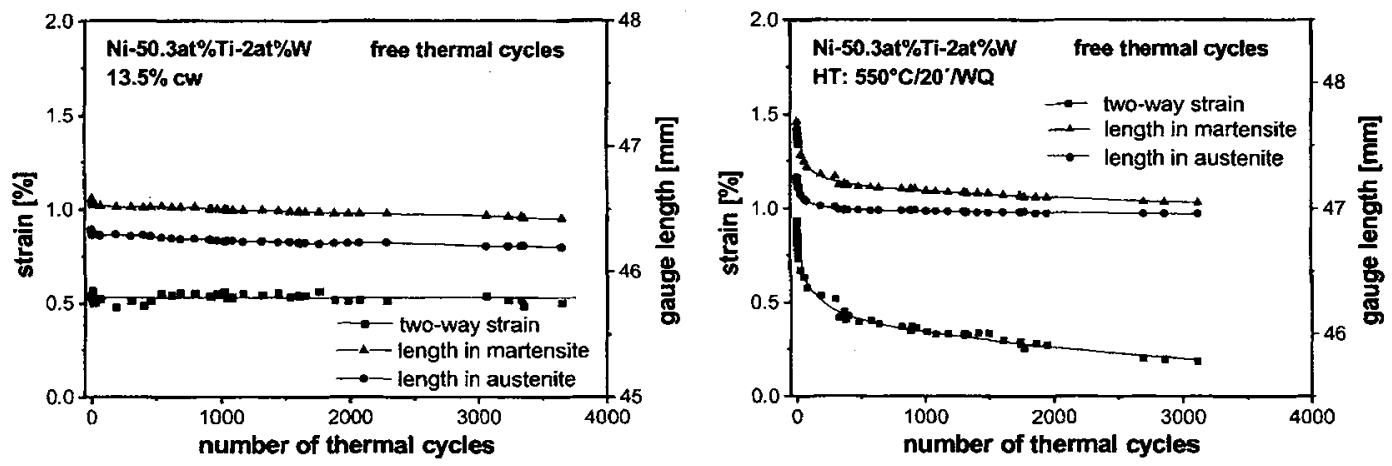

Figure 7: Change of the two-way effect due to thermal cycling; specimen B.
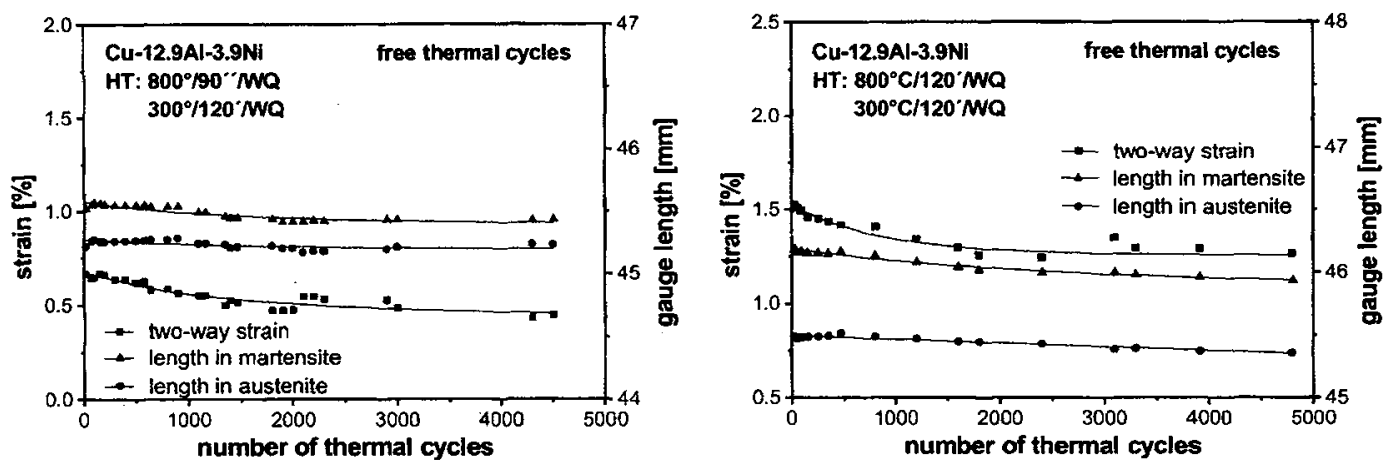

Figure 8: Change of the two-way effect due to thermal cycling, specimen $\mathrm{C}$.

Thus, the TWSME originates from stress fields caused by the microstructural features introduced during the thermomechanical training. However, since the biasing force is rather weak, its effect is considerably diminished if there exist large interfering internal stresses - as is the case in cold worked samples - leading to a less perfectly oriented martensitic structure. Consequently, as obvious from Figure 6 , the magnitude of the TWSME resulting from identical training treatments is smaller in the cold worked specimens compared to the annealed ones. However, a positive effect of cold working is observed regarding the stability of the effect. In both heat-treatment conditions a rather strong decay is evident in the initial stage of thermal cycling. This change of $\varepsilon_{2 w}$ in the early stage of cyclic application is ascribed to rearrangement and annihilation of dislocations until a new, saturated state is reached. With further cycling the degradation rate gradually decreases. As evident from Figure 6 , in the cold worked sample A1 the effect is almost stable in the latter stage, while it keeps degrading throughout the investigated range in the annealed specimen A2.

In contrast to the initial stage of thermal cycling, in the high cycle region the incomplete restoration of the high temperature shape $1^{\beta}$ is the major reason for the degradation of $\varepsilon_{2 w}$. Investigations of Perkins [6] showed, that dislocations emerge during thermally induced martensitic phase transformations in 
SMAs, even if no external stress is applied. An increase of the dislocation density leads to additional irreversible, true plastic strain, causing an increase of $1^{\beta}$ observable in Figure 6 . Comparing the two heattreatment conditions it becomes apparent that the decrease in the latter stage shows a sensitivity from the initial dislocation density. In the cold worked sample it is more difficult to generate additional dislocations as the dislocation density is already very high. This results in a more stable shape and accordingly to a more stable TWSME in the latter stage.

The conclusion that in the latter stage of fatigue cycling the degradation shows a sensitivity on cold working suggests that measures to improve the fatigue behaviour must aim at increasing the intensity of the internal stress field. Consequently, an approach is evaluated in this work by introducing Wdispersoids in alloy $\mathrm{B}$ and making use of the elastic stresses, resulting from the mismatch between the NiTi matrix and the dispersoids not taking part in the transformation. Figure 7 illustrates the change of the magnitude of the TWSME due to service cycles in alloy B. The mismatch created by the W-dispersoids causes elastic stresses stored in the matrix, reducing the effect of the microstructural features responsible for the TWSME. Consequently, the effect is smaller in the ternary alloy compared to alloy A. However, the stability of the effect during thermal cycling is actually improved. Especially in the cold worked condition alloy B exhibits an extraordinary stable behaviour. The element restores even after 4000 thermal cycles more or less the same shape in the austenite and martensite modification as in the first cycle.

Figure 8 summarizes the results of the experiments concerning the stability of the induced two-way strain in alloy $\mathrm{C}$. The degradation characteristics in CuAlNi are very different to those observed in the $\mathrm{NiTi}$ based ones. In the latter alloys the degradation curve was characterised by a rather sharp decay in the initial stage of thermal cycling and a gradual decrease of the two-way strain by further cycling, whereas in alloy $\mathrm{C}$ no dramatic change in the degradation rate is observed throughout the investigated period. In the fine grained specimens the effect shows a very stable behaviour and the dimensional stability $\left(1^{\beta}\right)$ is in both alloys far better than in NiTi. However, similar to the circumstances in alloys A and B, the improved stability is combined with a considerably smaller initial magnitude of the TWMSE.

\section{SUMMARY}

The degradation due to cyclic application is related to instabilities in the high- and low-temperature modification. The decrease of the effect size in NiTi-based alloys was found to consist of two characteristic stages, where the decay is distinguished by different origin and rate. In the first stage a strong decay is observed, which is mainly due to a loss of memory of the acquired low-temperature shape. In the high cycle range, on the other hand, the decrease is more gradual and it is attributed to a deterioration of the reversibility of the phase transformation. The stability of the effect can be considerably improved by the introduction of W-dispersoids. As shown with CuAINi, applying the same training treatment, a larger TWSME is achieved in a coarse grained than in a fine microstructure. The dimensional stability of the element is far better in CuAlNi compared to NiTi.

\section{Acknowledgements}

The authors gratefully acknowledge financial support of this work by the "Jubiläumsfond der österreichischen Nationalbank" (Austrian National Bank).

\section{References}

[1] H. Scherngell, A.C. Kneissl, Scripta Mat. 39, 205-212 (1998)

[2] H. Scherngell, A. Schnabl, A.C. Kneissl, Prakt.Met. Sonderband 30, 375-382 (1999)

[3] J. Perkins, R.O. Sponholz, Metall.Trans. 15A, 313-321 (1984)

[4] D. Ríos-Jara, G. Guénin, Acta metall. 35, 109-119 (1987)

[5] J. Perkins, Mat.Sci.Eng. 51, 181-192 (1981)

[6] J. Perkins, Metall.Trans. 4, 2709-2721 (1973) 